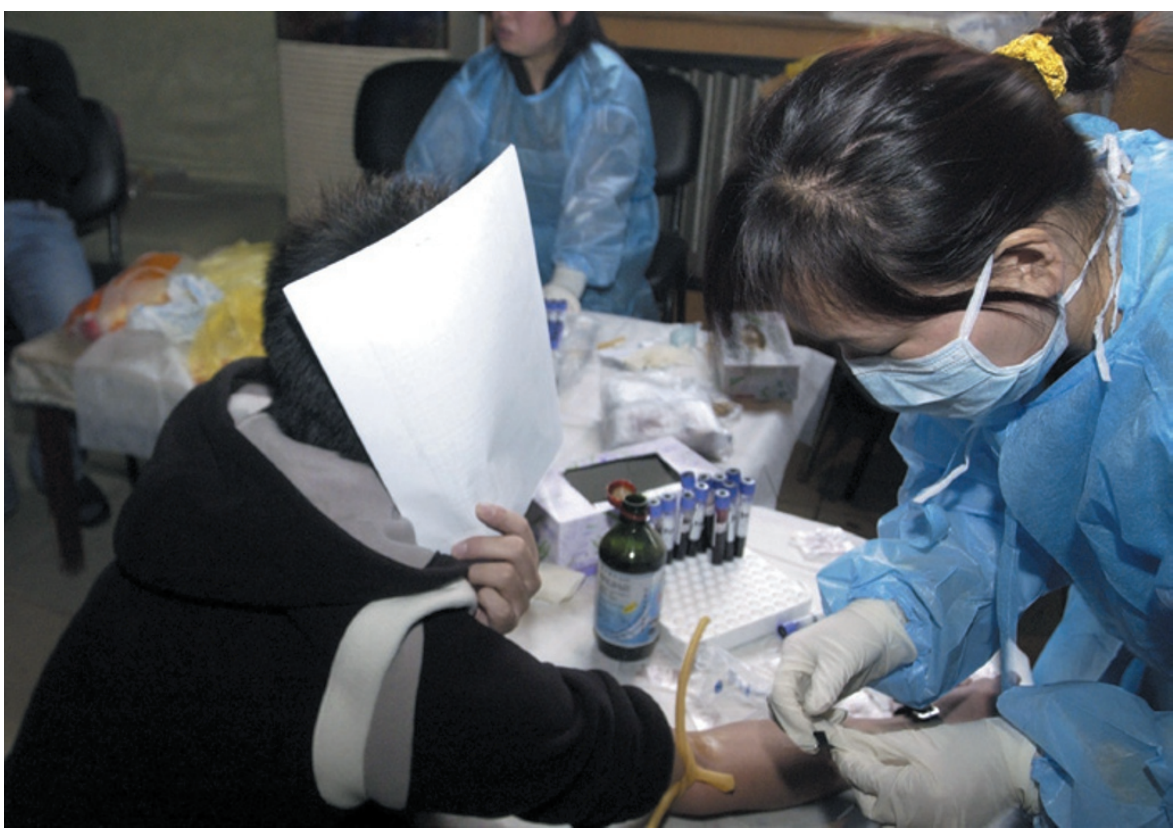

HIV testing is free in China, but many people fear having their HIV status exposed.

\title{
Bring safe sex to China
}

\section{China's discomfort over discussing sex, and especially homosexuality, is a major problem when it comes to $\mathrm{HIV}$, says a consortium of researchers in China.}

\section{A} n estimated 18 million men engage in homosexual sex in China, and they face growing risks to their health. Transmission between homosexuals has continued to gain ground among all reported HIV cases since surveillance of HIV among this group began a decade ago, rising from $0.3 \%$ before 2005 to more than $13.7 \%$ by 2011 (see 'On the rise').

The alarming spread of HIV among gay men is not seen in other high-risk groups, such as intravenous drug users and former commercial blood donors, and is fuelled by a stigma against homosexuality in China. In many ways, attitudes towards sexuality today in China resemble those of Western countries a quarter of a century ago. For instance, homosexuality was declassified as a mental illness in the United States in the early-1970s; this was not done in China until 2001. The main pressure for homosexuals to conceal their identity comes from friends and family, who place large emphasis on traditional obligations to uphold the family reputation and lineage. Many gay men say that they fear 'losing face', or having their social integrity damaged, if their orientation were to be revealed. Indeed, surveys of Chinese men who have sex with men have reported that up to $31.2 \%$ are married ${ }^{1}$.

Chinese people aren't uncomfortable just in discussing homosexuality — sex in general is still considered extremely personal and is rarely addressed openly or directly, irrespective of orientation. This discomfort has resulted in a pervasive stigma against people with HIV, a lack of general sex education for young people and poor epidemiological data about the spread of HIV in some populations around the country.

The result is a hidden population of individuals who are afraid to seek out HIV information resources or testing and counselling centres. Poorly educated, unaware of their HIV status and under pressure to conceal their sexual encounters, these men often engage in high-risk behaviour. And once one man hiding his activity becomes infected, the disease will spread among his partners, in an ongoing cycle. It is therefore no surprise that incidence of HIV has skyrocketed in this population.

This trend cannot continue. Policy-makers, public-health researchers, clinicians, educators, community leaders and other stakeholders in China must come together to educate everyone, and gay men in particular, about HIV prevention and treatment - before any more people become infected as a result of ignorance and fear.

The first outbreak of HIV was identified in China in the mid-1980s in intravenous drug users in southwestern provinces ${ }^{2}$. Over the past 20 years, it has not gained as strong a hold in China as in other countries: just $0.06 \%$ of the Chinese population is reportedly infected, less than one-fifth the prevalence in the United States and Europe (and one-hundredth that of sub-Saharan Africa) ${ }^{3}$.

In 2003, under international criticism and a growing awareness of the potential impact of a generalized epidemic, the Chinese government developed a systemized HIV prevention and treatment programme. The resultant 'Four Frees and One Care' act provides free access to antiretroviral drugs to people with AIDS living in rural areas, poor people living in urban areas and pregnant woman; free counselling and testing for all; free schooling for orphans of parents with AIDS; and care and economic help for the families of people living with HIV/AIDS. But many people say that they are not aware of the provisions, or of how to find free testing centres ${ }^{4}$.

Although the government supports condoms as the primary means of preventing disease transmission, public advertisement campaigns promoting their use have been limited until the past few years. This is due, in part, to the absence of general sex education programmes in the vast majority of Chinese schools - a gap that is often left open by parents who aren't comfortable discussing sex with their children. This fundamental lack of knowledge has huge implications for combating HIV, not least because it carries over to health-care professionals. Even if people work up the courage to ask about HIV testing and information, health-care workers may not have adequate training, or be comfortable discussing the sexual conduct associated with HIV and other sexually transmitted diseases. This, coupled with stigma that leads men to hide their homosexual activity, means that men delay HIV testing and treatment — and, as a result, put all their partners at risk.

\section{CAUSE FOR CONCERN}

Although both international and national groups have identified a rise in new infections in homosexual men in China, precise monitoring of the disease remains one of the greatest hurdles facing HIV researchers. It was not until 2010 that the first large-scale government surveillance results were released. This study of 26,000 Chinese men who engage in homosexual activity in 61 cities, conducted from 2008 to 2009 , showed that average HIV prevalence was as high as $5 \%$, and more than $10 \%$ in cities where the epidemic was first identified $^{5}$. Unfortunately, national survey data tend to be published in broad terms, with limited source identification, making it difficult to evaluate research methodologies.

If these figures are correct, then homosexual men represent just $13 \%$ of HIV cases - a much smaller percentage than in the United States, where the number is 
around 50\%. Although gay men are still the minority of HIV cases in China, behind drug users, blood donors and people infected through heterosexual sex, the growing proportion of cases among gay men is alarming. Before 2005, homosexual transmission was claimed to represent just $0.3 \%$ of cases $^{6}-$ less than $1 / 45$ th its proportion today. At this rate, the picture in China will soon resemble that in the United States. Like in the United States, the genetic diversity of the viruses circulating in the Chinese population continues to increase as a result of multiple introduction $^{7}$ and de novo generation of recombinant strains ${ }^{8}$, many of which are resistant to one or more antiretroviral drugs, creating further challenges for effective treatment and vaccine development.

The rapid rise in new infections in homosexual men in China is fuelled by high rates of high-risk behaviour ${ }^{4}$. Around $40 \%$ of homosexual men there have exchanged money for sex. In some places, such as Liaoning province, drug use is significantly correlated with disease prevalence. Homosexual men are also among China's 145 million migrants - a byproduct of China's rapid economic development - a lifestyle that research shows goes hand in hand with unprotected sex, exchanging sex for money and limited knowledge of $\mathrm{HIV}^{4}$. Such high-risk behaviour makes men who have sex with men significantly more vulnerable to infection, and creates an open door for generalized disease transmission.

\section{TIME TO ACT}

China is not the only nation struggling to control HIV among all at-risk groups, but we believe that these barriers to prevention and treatment can be overcome, around the world, through a cooperative effort between the government, health professionals and public programmes. Such an effort will require an environment in which individuals are well informed on why and how to protect themselves, and are not afraid to seek healthcare resources. The key factor, ultimately, lies in breaking through stigmas about sex, and about homosexuality and HIV in particular.

First, at the political level, it is important to highlight the remarkable progress that has been made in China over the past ten years in terms of government support for HIV research, prevention and treatment for homosexuals, which has been commended by the World Health Organization, UNAIDS and other global health groups ${ }^{9}$. Efforts to combat the stigma, however, have been insufficient. In 2006, the Chinese government came up with recommendations to eliminate legal and public prejudices against infected individuals, but these have yet to be turned into clear legal guidelines. Policy-makers must make discrimination against HIVpositive individuals punishable by law, and include sufficient incentives or penalties to ensure that the policies are enforced. We also believe that legislation should include punishments for knowingly exposing another person to HIV, and requirements for HIVinfected patients to notify their physician and partners when they are diagnosed with HIV.

Second, health professionals, including researchers and medical workers, must cooperate across disciplines to better characterize where and how HIV is gaining ground, and to translate these data into public-health applications. To start, more HIV testing, and better prevention and treatment interventions are needed, especially because antiretroviral chemoprophylaxis ${ }^{10}$ and treatment ${ }^{11}$ can significantly reduce transmission. Until more homosexual men understand how to access these services, these communities need to be targeted with testing and counselling, including the importance of knowing their status.

Public programmes and grass-roots efforts are the foundation of HIV prevention. Policy changes, therefore, must go hand-in-hand with efforts to increase public education

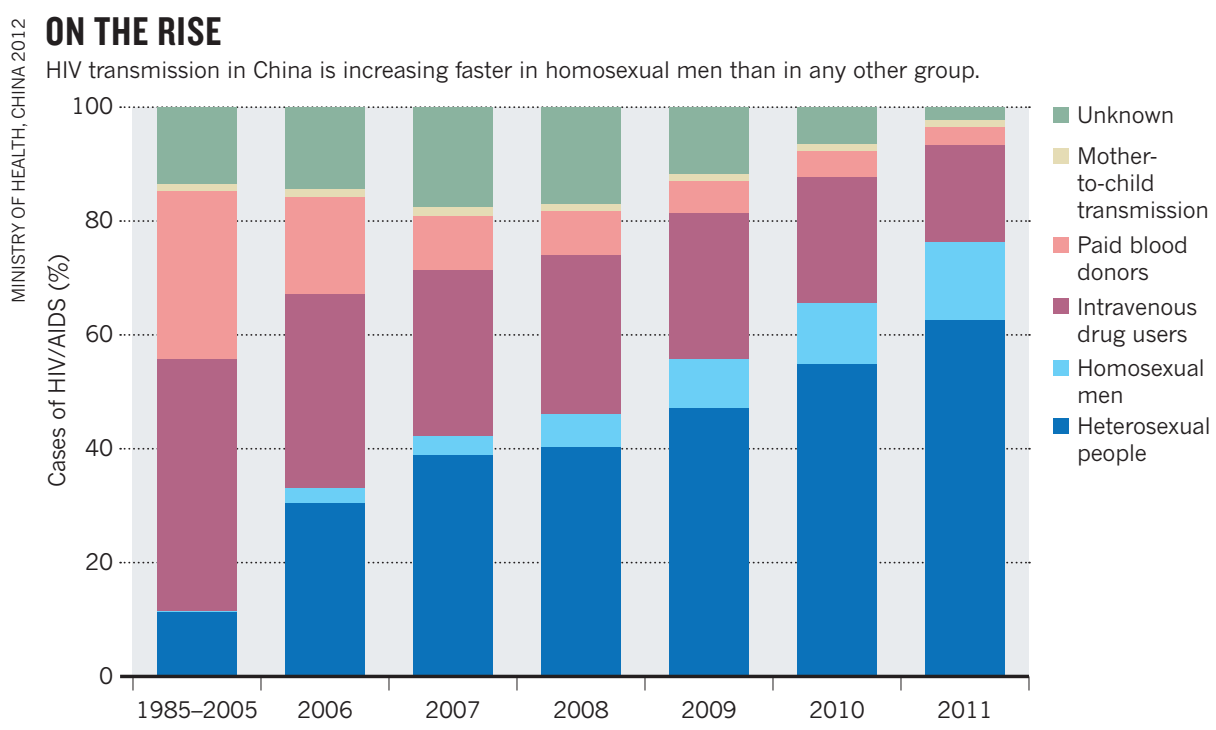

and awareness of HIV, specifically targeting homosexual and migrant populations. Health campaigns should embrace digital media, and target the online chat rooms and websites frequently accessed by homosexual men, promoting condom use and reliable resources for HIV and general sex education.

Leading by example is one of the most powerful ways to combat stigma. In the Western world, the first brave individuals to reveal that they had HIV caused a significant shift in public thinking about the disease. In 2004, basketball legend Magic Johnson and native-Chinese basketball player Yao Ming produced a series of public-service announcements to combat HIV discrimination that were widely broadcast in China. In 2003, a man stood up during an AIDS summit with former US President Bill Clinton at Tsinghua University in Beijing, and in doing so became one of the first Chinese people to publicly reveal his HIV status. Clinton embraced the man, named Peng-fei Song, bringing him positive media attention. Song later became a strong advocate for HIV awareness and prevention. China needs to encourage more individuals affected by HIV/AIDS to step up and help to change people's perception of the disease.

Only through a large-scale, coordinated effort between policy, programme, research and clinical sectors will new infections start to significantly decrease. The challenge is not insurmountable. But it does require concerted action - now.

Hong Shang, Junjie Xu and Xiaoxu Han are at the Key Laboratory of AIDS Immunology of Ministry of Health, Department of Laboratory Medicine, No. 1 Hospital of China Medical University, Shenyang 110001, China. Jessica Spero Li, Kelly C. Arledge and Linqi Zhang are at the Comprehensive AIDS Research Center and Research Center for Public Health, School of Medicine, Tsinghua University, Beijing, 100084, China.

e-mail:hongshang100@hotmail.com or zhanglinqi@tsinghua.edu.cn

1. Yun, K. et al. Sex. Transm. Infect. 87, 563-570 (2011)

2. Lu, L. et al. Nature $455,609-611$ (2008).

3. UNAIDS Report on the Global AIDS Epidemic (UNAIDS, 2010).

4. Song, Y. et al. AIDS Care 23, 179-186 (2011).

5. Chinese Ministry of Health China 2010 UNGASS Country Progress Report (2008-2009) (Ministry of Health of the People's Republic of China, 2010).

6. Chinese Ministry of Health 2012 China AIDS Response Progress Report (Ministry of Health of the People's Republic of China, 2012).

7. Zhang, Y. et al. PLoS Med. 3, e443 (2006).

8. Shang, H. et al. J. Biol. Chem. 286, 14531-14541 (2011).

9. Wu, Z. Sullivan, S. G., Wang, Y., Rotheram-Borus, M. J. \& Detels, R. Lancet 369, 679-690 (2007).

10.Grant, R. M. et al. N. Engl. J. Med. 363, 2587-2599 (2010).

11.Cohen, M. S. et al. N. Engl. J. Med. 365, 493-505 (2011). 